\title{
Krawtchouk Moments for Gait Phase Detection
}

\author{
C. Camacho-Bello and J.J. Báez-Rojas \\ Instituto Nacional de Astrofísica, Óptica y Electrónica, Luis Enrique Erro No. 1, \\ 72840, Puebla, México
}

\begin{abstract}
We present a novel method for gait phase detection based on Krawtchouk moments, which can be used in gait analysis. The low computational cost and high capacity of description of the Krauchouk moments makes it easy detect the parameters of the gait cycle, such as the swing phase, stance phase and double support. In addition, we present the results of the gait phases detection with the proposed method of 10 test subjects and compared with standard values.
\end{abstract}

Keywords: Krawtchouk moments, gait analysis, gait phase detection.

\section{Introduction}

The human gait is a complex mechanism by which a variety of muscles work together in coordination to move from one place to another, with low-effort and minimum energy consumption. Given the high complexity that is presented for gait analysis, it usually described in terms of periods and phases, to facilitate the description and study. In this sense, the gait cycle is the period of time between any two identical events in the walking cycle [1]. Figure 10 shows the temporal components of the different supports each foot during the gait cycle. In normal walking, the gait cycle is comprised of $62 \%$ stance phase and $38 \%$ swing phase with two periods of double support that occupy a total of $25 \%$ of the gait cycle. Abnormal measures of temporal parameters have proved to be important indicators of gait dysfunction [2]. The gait analysis is useful for documenting patient progress and for doing clinical research.

The study of human gait with the technique of orthogonal moments has been studied in different contexts, such as gait recognition [3-6] and moving objects reconstruction [7]. An advantage of using orthogonal moments to extract features of the shape and motion information is that it requires few descriptors [3]. Recently, Ioannidis et al. [4] used the Krawtchouk moments and Radon transformation for gait recognition. However, they only extracted descriptors that are contained in the gait cycle and do not analyze the gait phases. Moreover, Camacho et al. [6] proposed a method based on Jacobi-Fourier moment history for gait recognition and mentions that there is a relationship between the moment history and the gait phases, but do not calculate the temporal parameters and percentages of gait phases. The continuous orthogonal moments defined inside the unit circle as presented in previous work, require a coordinate transformation and suitable approximation of the integration of moment kernel, unlike the

E. Bayro-Corrochano and E. Hancock (Eds.): CIARP 2014, LNCS 8827, pp. 7877793 2014.

(C) Springer International Publishing Switzerland 2014 
discrete orthogonal moments that are directly defined in the image coordinate space and preserve the property of orthogonality [8], of which the most prominent are the Tchebichef moments, the Hahn moments and the Krawchouk moments. The latter present a good results in the extraction of features [9 11], because of its ability to extract local features of an image, with minimum redundant information.

In this paper, we present a novel method based on the analysis of a time series of Krawtchouk moments and the mean value between the maximum and minimum of the time series, which facilitates the calculation of the phases of gait, cadence and time a person to take a step. We also present the results of the percentages of the phases of gait and temporal parameters of 10 test subjects.

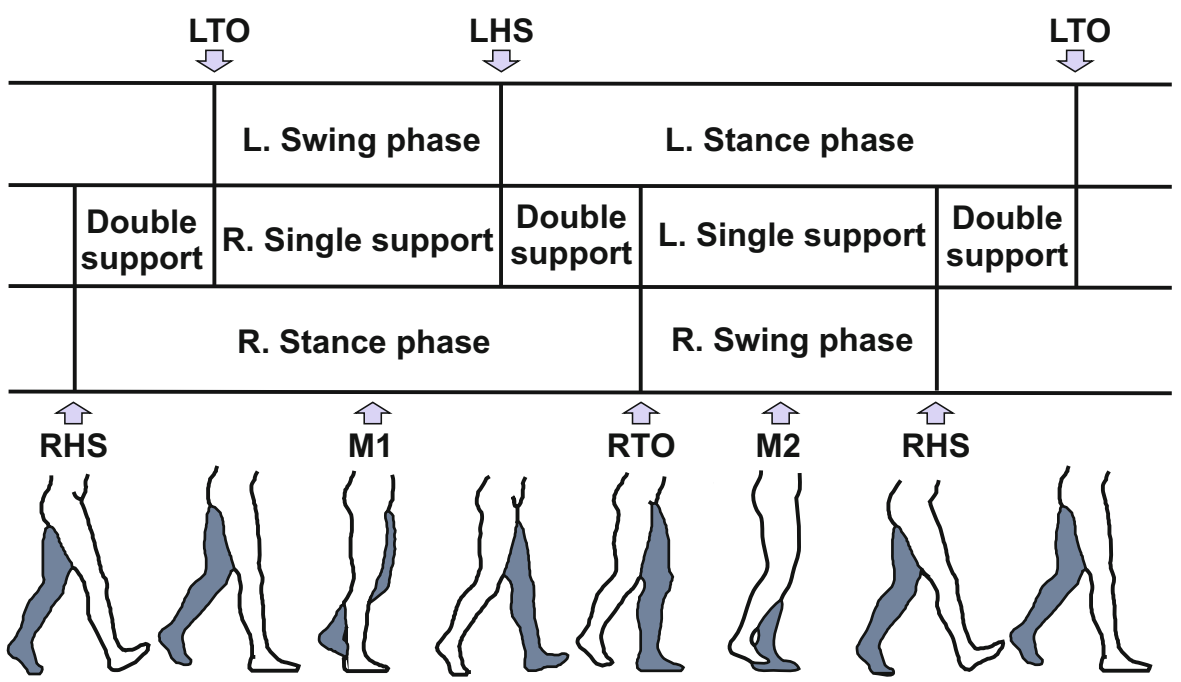

Fig. 1. Different phases of gait cycle. RHS: Right heel strike; RTO:right toe-off; LHS: left heel strike; LTO: left toe-off; M1:mid stance; M2: mid swing.

\section{Krawtchouk Moments}

Yap et al. 9] proposed the Krawtchouk moments for image analysis, where the weighted and normalized Krawtchouk polynomials are used as a basis set, which is defined by,

$$
\widetilde{k}_{n}(x ; p, N)=k_{n}(x ; p, N) \sqrt{\frac{w(x ; p, N)}{\rho(n ; p, N)}}
$$

where $w(x ; p, N)$ is the weighted function, $\rho(n ; p, N)$ is the norm, and $k_{n}(x ; p, N)$ are the Krawtchouk polynomials. The polynomials $\widetilde{k}_{n}(x ; p, N)$ satisfies the orthogonality property is given by, 


$$
\sum_{x=0}^{N-1} \widetilde{k}_{n}(x ; p, N) \widetilde{k}_{m}(x ; p, N)=\delta_{n, m}
$$

where $\delta_{n, m}$ is the Kronecker symbol. In order to improve the calculation efficiency of Krawtchouk polynomials, we propose a recurrence relation with respect to $n$, which has a smaller number of operations that some of the proposals recurrence relations [9] [12] [13]. The recurrence relation for Krawtchouk polynomials is given by,

$$
A_{n} \widetilde{k}_{n}(x ; p, N)=B_{n} \widetilde{k}_{n-1}(x ; p, N)-A_{n-1} \widetilde{k}_{n-2}(x ; p, N),
$$

where

$$
\begin{aligned}
& A_{n}=\sqrt{p(1-p)(N-n+1) n} \\
& B_{n}=x-n+1-p(N-2 n+2)
\end{aligned}
$$

For the initial numerical calculation, the Krawtchouk polynomials of the zeroth and first normalized orders are given by

$$
\begin{aligned}
\widetilde{k}_{0}(x ; p, N) & =\sqrt{w(x ; p, N)} \\
\widetilde{k}_{1}(x ; p, N) & =(x-p N) \widetilde{k}_{0}(x ; p, N)
\end{aligned}
$$

where $w(x ; p, N)$ can be calculated recursively using

$$
w(x ; p, N)=\frac{p(N-x+1)}{(1-p) x} w(x-1 ; p, N)
$$

with $w(0 ; p, N)=(1-p)^{N}$. The recurrence relations with respect to $n$ cause numerical instability for higher orders to reduce this problem proposes using recurrence relations with respect to $x$ 12] [8], unfortunately also causes numerical instability for images with $N$ large [13]. In this paper, we only use low order polynomials for the calculation of the orthogonal moments, which provide global information of the image to be analyzed.

The Krawtchouk moments of order $(n+m)$ in terms of weighted Krawtchouk polynomials, for an image with intensity function, $f(x, y)$, is defined as

$$
Q_{n m}=\sum_{x=0}^{N-1} \sum_{y=0}^{M-1} \widetilde{k}_{n}\left(x ; p_{x}, N\right) \widetilde{k}_{m}\left(y ; p_{y}, M\right) f(x, y) .
$$

By varying the parameters $p_{x}$ and $p_{y}$ of the binomial distribution associated with Krawtchouk polynomials may be able to extract local features from any region-of-interest in an image. For two-level images used in this paper, we can find the area of interest using geometric moments, which are defined using the discrete approximation as

$$
M_{n m}=\sum_{x=0}^{N-1} \sum_{y=0}^{M-1} x^{n} y^{m} f(x, y) .
$$


The centroids for the parameters $p_{x}$ and $p_{y}$ are given as follows,

$$
\begin{aligned}
& p_{x}=M_{1,0} / M_{00}, \\
& p_{y}=M_{0,1} / M_{00} .
\end{aligned}
$$

One way to test the ability of local features extracting with the Krawtchouk moments is through image reconstruction, which is calculated as follows,

$$
\widetilde{f}(x, y)=\sum_{n=0}^{L_{x}} \sum_{m=0}^{L_{y}} \widetilde{k}_{n}\left(x ; p_{x}, N\right) \widetilde{k}_{m}\left(y ; p_{y}, M\right) Q_{n m} .
$$

where $\tilde{f}(x, y)$ is the reconstructed image, $L_{x}$ and $L_{y}$ are the maximum order of reconstruction. Figure 2 shows the reconstruction of an image of a person walking with $p_{x}=0.29$ and $p_{x}=0.5$, which were obtained from Eqs. 11 and 12 respectively. Note that for the reconstruction of low-order $\left(L_{x}=2,10\right)$ with $p_{x}=p_{y}=0.5$ are not able to reconstruct the image of a person walking, unlike those calculated with Eqs. 11 and 12.

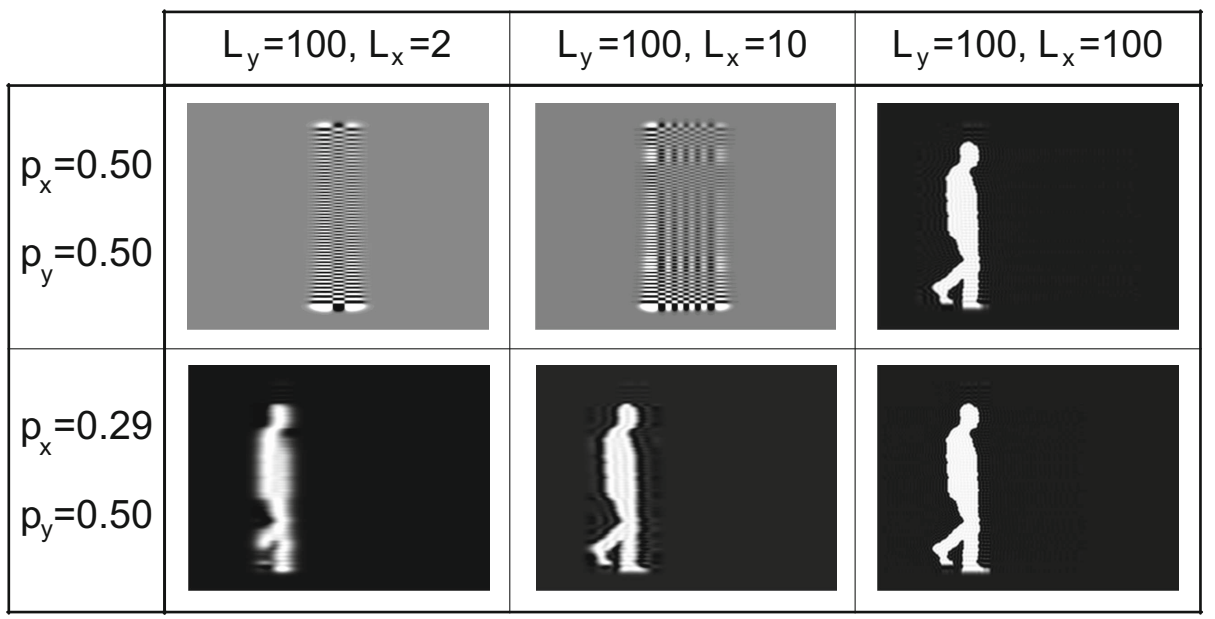

Fig. 2. Reconstructed image of a person walking with different values of $L_{x}$ and $p_{x}$

\section{The Gait Phase Detection Algorithm}

As is well known the low-order moments are associated with the form, while the high-order moments of the image detail [14]. Therefore, the low-order moments can be used for time series analysis from a set of images to describe the movement over time of an object or a person performing a physical activity. In the case of human gait analysis can extract information about temporal parameters, such as the swing phase, stance phase, double support and cadence. Figure 3 shows 


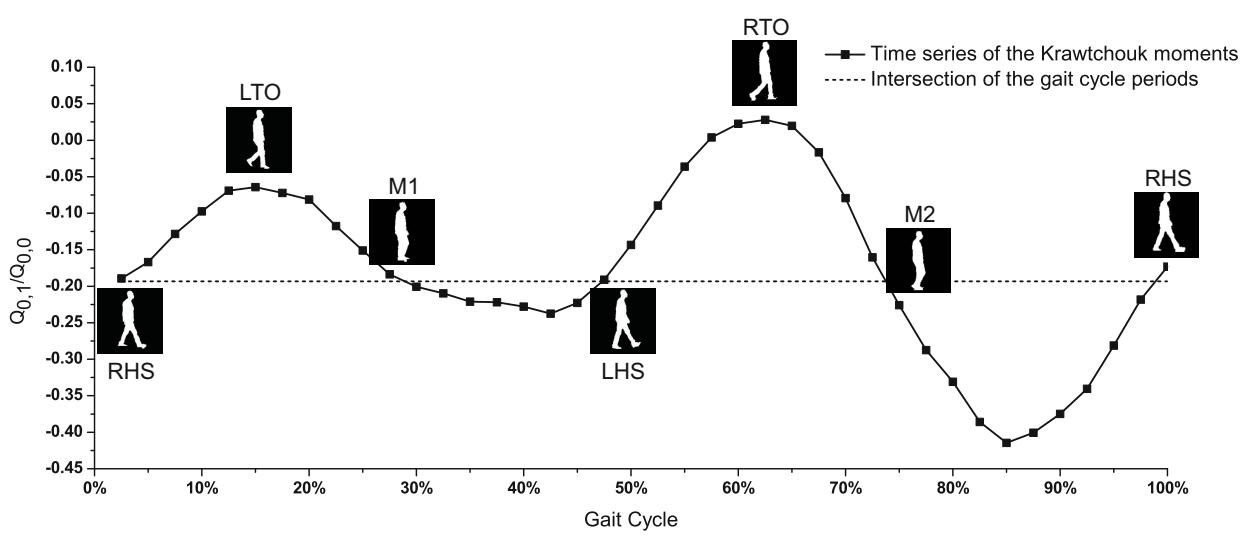

Fig. 3. Gait cycle associated with Krawtchouk moments

the gait cycle obtained with Krawtchouk moments of low-order normalized by zero-order moments.

To facilitate the location of gait phases in the time series of Krawtchouk moments, we propose obtaining these parameters from a line that intercepts the different gait phases, which is given by

$$
g=\frac{\max Q_{n, m}(t)+\min Q_{n, m}(t)}{2}
$$

where $Q_{n, m}(t)$ are the Krawtchouk moments of an image at a time $t$. The line is given by the value of $g$ intersects phases: right heel strike (RHS), left heel strike (LHS), mid stance (M1) and mid swing (M2). While the phases of left toe-off (LTO) and right toe-off (RTO) can be found by the maximum of the time series, all this can be seen in Fig. 3 .

By identifying the gait cycle in the time series can be calculated by temporal parameters, it is only need to know the speed of the video camera. For example, in Fig. 3, the gait cycle is completed in 39 images taken at a rate of 30 frames per second, therefore the gait cycle lasts $1.3 \mathrm{~s}$. In the same way, one can calculate the time of a step, stance phase, swing phase, double support and cadence.

\section{Experimental Results}

The experimental study compares average values of the temporal parameters obtained with 10 test subjects with the proposed method and the standard of normal gait in male adult of the Mexican population. The subjects met the following criteria: male; between 18 and 40 years; weight within 65 to $82 \mathrm{~kg}$. ; absence of any pathology of bone or muscle. The percentages of the phases of the gait cycle are standard values. However, temporal parameters have variations due to anthropometric differences between populations from different countries; therefore, we compared the temporal parameters with the results obtained by 
Table 1. Temporal parameters of gait cycle

\begin{tabular}{lcccc} 
& \multicolumn{2}{c}{ Pérez-Orive et al. } & 15] & Proposed method \\
& Average & Std. & Average & Std. \\
\hline Gait cycle & $1.14 \mathrm{~s}$ & $0.07 \mathrm{~s}$ & $1.23 \mathrm{~s}$ & $0.07 \mathrm{~s}$ \\
Step & $0.57 \mathrm{~s}$ & $0.04 \mathrm{~s}$ & $0.60 \mathrm{~s}$ & $0.04 \mathrm{~s}$ \\
Stance phase & $0.64 \mathrm{~s}$ & $0.05 \mathrm{~s}$ & $0.75 \mathrm{~s}$ & $0.06 \mathrm{~s}$ \\
Swing phase & $0.50 \mathrm{~s}$ & $0.03 \mathrm{~s}$ & $0.47 \mathrm{~s}$ & $0.04 \mathrm{~s}$ \\
Double support & $0.07 \mathrm{~s}$ & $0.02 \mathrm{~s}$ & $0.30 \mathrm{~s}$ & $0.03 \mathrm{~s}$ \\
\hline
\end{tabular}

Pérez-Orive et al. [15] for Mexican adult male. Table 1 shows standard values and the calculated using Krawtchouk moments.

The results collaborate the standard values Pérez-Orive et al. [15], which reports that the duration of the gait cycle is slightly higher than reported. Moreover, the proposed method can calculate the percentages of the gait cycle, the values obtained are the following: $61 \%$ stance phase, $39 \%$ swing phase, and $24 \%$ double support.

\section{Conclusions}

In conclusion, we propose a new method for gait phase detection using Krawtchouk moments, which reliably identify the transitions between the phases of stance, swing, right heel strike, right toe-off, left heel strike, left toe-off, mid stance, and mid swing. The proposed method is easy to implement and has a low computational cost, because a single descriptor can find the gait phases. In addition, significantly improves the quality of descriptor using the centroids of the image for parameters $p_{x}$ and $p_{y}$ of the Krawtchouk moments. The results obtained for the duration of the gait parameters are relatively higher than others reported for different populations, as reported by Pérez-Orive et al. [15]. However, percentages calculated with the Krawtchouk moments correspond to the standard values, unlike percentages by Pérez-Orive et al. [15] for Mexican adult male population. We believe that the proposed method will serve for human gait analysis and results presented can contribute to the rehabilitation of patients with some condition of walking.

\section{References}

1. Ayyappa, E.: Normal Human Locomotion, Part 1: Basic Concepts and Terminology. Journal of Prosthetics and Orthotics 9(1), 10-17 (1997)

2. Davis, R.B.: Clinical gait analysis. IEEE Eng. Med. Biol. Magazine 7, 35-40 (1998)

3. Shutler, J.D., Nixon, M.S.: Zernike velocity moments for sequence-based description of moving features. Image and Vision Computing 24, 343-356 (2006)

4. Ioannidis, D., Tzovaras, D., Damousis, I.G., Argyropoulos, S., Moustakas, K.: Gait Recognition Using Compact Feature Extraction Transforms and Depth Information. IEEE Transactions on Information Forensics and Security 2, 623-630 (2007) 
5. Camacho-Bello, C., Toxqui-Quitl, C., Padilla-Vivanco, A.: Gait Recognition by Jacobi-Fourier moments. In: FIO/LS Technical Digest OSA (2011)

6. Camacho-Bello, C., Toxqui-Quitl, C., Padilla-Vivanco, A.: Generic Orthogonal Moments and Applications. In: Papakostas, G.A. (ed.) GCSR, vol. 1, pp. 175-204. Science Gate Publishing (2014)

7. Prismall, S.P., Nixon, M.S., Carter, N.: On moving object reconstruction by moments. In: Proceedings of the 13th British Machine Vision Conference, pp. 83-82 (2002)

8. Mukundan, R.: Some computational aspects of discrete orthonormal moments. IEEE Transactions on Image Processing 13, 1055-1059 (2004)

9. Yap, P.T., Paramesran, R., Ong, S.H.: Image analysis by Krawtchouk moments. IEEE Trans. Image Process. 12, 1367-1377 (2003)

10. Hu, B., Liao, S.: Chinese Character Recognition by Krawtchouk Moment Features. In: Kamel, M., Campilho, A. (eds.) ICIAR 2013. LNCS, vol. 7950, pp. 711-716. Springer, Heidelberg (2013)

11. Sheeba Rani, J., Devaraj, D.: Face recognition using Krawtchouk moment. Sādhanā 37, 441-460 (2012)

12. Zhu, H., Liu, M., Shu, H., Zhang, H., Luo, L.: General form for obtaining discrete orthogonal moments. IET Image Processing 4, 335-352 (2010)

13. Zhang, G., Luo, Z., Fu, B., Li, B., Liao, J., Fan, X., Xi, Z.: A symmetry and birecursive algorithm of accurately computing Krawtchouk moments. Pattern Recognition Letters 4, 548-554 (2010)

14. Padilla-Vivanco, A., Urcid-Serrano, G., Granados-Agustín, F., Cornejo-Rodríguez, A.: Comparative analysis of pattern reconstruction using orthogonal moments. Opt. Eng. 46, 017002 (2007)

15. Pérez-Orive, J., Pichardo, A.E., Chávez-Arias, D.: Desarrollo de un estándar de marcha normal en hombres adultos. Rev. Mex. Ortop. Traum. 12 (1998) 